\title{
BET_VH: exploring the influence of natural uncertainties on long-term hazard from tephra fallout at Campi Flegrei (Italy)
}

\author{
Jacopo Selva • Antonio Costa • Warner Marzocchi • \\ Laura Sandri
}

Received: 4 December 2008 / Accepted: 9 February 2010 / Published online: 18 March 2010

(C) The Author(s) 2010. This article is published with open access at Springerlink.com

\begin{abstract}
In this paper, we explore the effects of the intrinsic uncertainties upon long-term volcanic hazard by analyzing tephra fall hazard at Campi Flegrei, Italy, using the BET_VH model described in Marzocchi et al. (Bull Volcanol, 2010). The results obtained show that volcanic hazard based on the weighted average of all possible eruptive settings (i.e. size classes and vent locations) is significantly different from an analysis based on a single reference setting, as commonly used in volcanic hazard practice. The long-term hazard map for tephra fall at Campi Flegrei obtained here accounts for a wide spectrum of uncertainties which are usually neglected, largely reducing the bias intrinsically introduced by the choice of a specific reference setting. We formally develop and apply a general method to recursively integrate simulations from different models which have different characteristics in terms of spatial
\end{abstract}

Editorial responsibility: R. Cioni

J. Selva $\cdot$ L. Sandri

Istituto Nazionale di Geofisica e Vulcanologia,

Bologna, Italy

\section{A. Costa}

Osservatorio Vesuviano, Istituto Nazionale di Geofisica

e Vulcanologia, Napoli, Italy

A. Costa

Department of Earth Sciences, University of Bristol,

Bristol, UK

W. Marzocchi $(\varangle)$

Istituto Nazionale di Geofisica e Vulcanologia,

Rome 1, Italy

e-mail: warner.marzocchi@ingv.it coverage, resolution and physical details. This outcome of simulations will be eventually merged with field data through the use of the BET_VH model.

Keywords Volcanic hazard • Ash fall • Bayesian event tree $\cdot$ Campi Flegrei

\section{Introduction}

A reliable probabilistic volcanic hazard assessment (PVHA) is the basic scientific component for planning long-term risk mitigation actions. Despite its importance, we argue that very few efforts have been made to verify the influence of the many natural uncertainties on the final assessment. Here, we explore this point, with particular emphasis on the effects of the variability in eruption size class (with this term, we mean any kind of parametrization of the magnitude, intensity, or type of eruption; see Marzocchi et al. 2010) and vent location upon PVHA. This variability represents an objective difficulty in achieving a reliable PVHA. Indeed, PVHA is usually based on single eruptive scenarios analysis (e.g. Barberi et al. 1995, 2008; DPC 1995), on comparison among single scenarios (e.g. Costa et al. 2009), and/or analysis of phenomena which have occurred in the past (e.g. Orsi et al. 2004). To date, most PVHA applications are based on the analysis of single scenarios selected as the most probable or the most representative. In this way, the aleatory variability associated with the uncertainty in terms of the size class and vent locations is completely ignored.

The single scenario approach is often assumed because of the difficulty in merging together, in a consistent way, the information from different "eruptive 
settings" (ESs), defined as the occurrence of an eruption of a specific size class from a vent in a specific location (see Marzocchi et al. 2010). This is a crucial issue since both central volcanoes and calderas may erupt with wide variability in terms of size of the eruption and location of the vent (e.g. Simkin and Siebert 1994). In short-term applications, some attempts to reduce such variability have been adopted (e.g. Lindsay et al. 2010). In long-term applications, as in the present study, it is practically unavoidable, given that nowadays, it is impossible to "predict" the size and vent location of the next eruption.

In this paper, we consider the case of long-term tephra fall hazard at Campi Flegrei. Campi Flegrei caldera $(\mathrm{CFc})$ is a highly urbanized area that has experienced significant variability both in vent location and size of eruptions (e.g. Orsi et al. 2004). In practice, we analyze the effects of the inclusion of different ESs and other uncertainties on the hazard posed by tephra fall at $\mathrm{CF}$ c using the Bayesian Event Tree for Volcanic Hazard (BET_VH) code described in the companion paper (Marzocchi et al. 2010). In this way, we can also directly check the reliability of PVHA based on single scenarios.

Furthermore, in this paper, we propose and apply a scheme that formally merges information coming from different models and field data relative to a generic volcanic outcome (e.g. pyroclastic flow, lava flow, tephra fall, lahars etc.) into the BET_VH model. The BET_VH model (Marzocchi et al. 2010) is a formal Bayesian inference procedure to assess volcanic hazard, based on an event tree schema. While the use of models, theoretical beliefs, past data etc., relative to eruption forecasting, has been widely discussed in previous papers (e.g. Marzocchi et al. 2008; Sandri et al. 2009), the inference of the long-term hazard posed by a generic volcanic outcome has not yet been treated. In this paper, we present a scheme that integrates the output of models with different reliability and coverage in order to provide a single prior probability distribution associated with each selected volcanic outcome. In this scheme, the output of each model is included by weighting it according to the reliability of the model itself. For instance, simple models can be used to provide a full description of all possible ESs and relative initial-boundary conditions; this is usually possible because simple models are computationally very fast, and thus, many simulations can be performed. On the other hand, more sophisticated models, characterized by longer computational times, may be used to better define a single ES of particular interest, such as the most likely ES. In this way, the prior distribution may be more precise for some specific ESs, reducing the epistemic uncertainty as much as possible. At the same time, it may provide an unbiased assessment of any other realistic possibility. Eventually, such a prior distribution may be then merged with field data collected from past eruptions in order to provide a full picture of the hazard posed by the selected volcanic outcome (posterior distribution).

To summarize, here we apply the BET_VH model to long-term tephra hazard assessment at the $\mathrm{CFc}$ with two goals: (1) to explore the effects of the uncertainty in size class and vent locations on the final assessment and (2) to provide a tutorial example of how the outputs of tephra dispersion models can be embedded into BET_VH. We emphasize that this tutorial example can be generalized in a straightforward fashion to any other kind of phenomena, such as pyroclastic flows, lava flows, or lahars, and to any other volcano.

\section{From simulation output to prior distribution parameters at nodes 7 and 8 in BET_VH}

The model BET_VH (Marzocchi et al. 2010) enables us to achieve a full volcanic hazard assessment for each generic volcanic outcome (e.g. pyroclastic flow, lava flow, tephra fall, lahars etc.). The Bayesian inference scheme applied to an event tree is schematized in Fig. 1a (see Marzocchi et al. 2010).

In this section, we describe how to implement the prior distribution relative to nodes 7 and 8 . In practice, at such nodes, BET_VH describes the diffusion process of a selected volcanic outcome, given that an eruption (nodes 1-2-3) in a specific vent (node 4) with a specific size class (node 5) has produced the outcome (node 6). More specifically, at node 7 , the possibility that a given area is reached by the selected volcanic outcome is estimated; we call this possibility "conditional probability of reaching episodes". At node 8, the possibility that, in the same area considered at node 7 , the volcanic outcome overcomes a specific threshold is estimated; we call this possibility "conditional probability of overcoming episodes".

In order to define the prior probabilities at nodes 7 and 8, we need mathematical models that describes the spatial dispersion of the selected outcome in the volcano surrounding areas. In a second step, these prior probabilities may be eventually combined with field data (see Marzocchi et al. 2010). In "Appendix", we develop a method to convert the output of one or more theoretical models into the parameters of the prior distribution. Here, we report the final equations only; further details can be found in "Appendix". To help readers, in Tables 1 and 2, we report a complete 
Fig. 1 Event tree of the model BET_VH (a) for a generic volcano and (b) for Campi Flegrei a

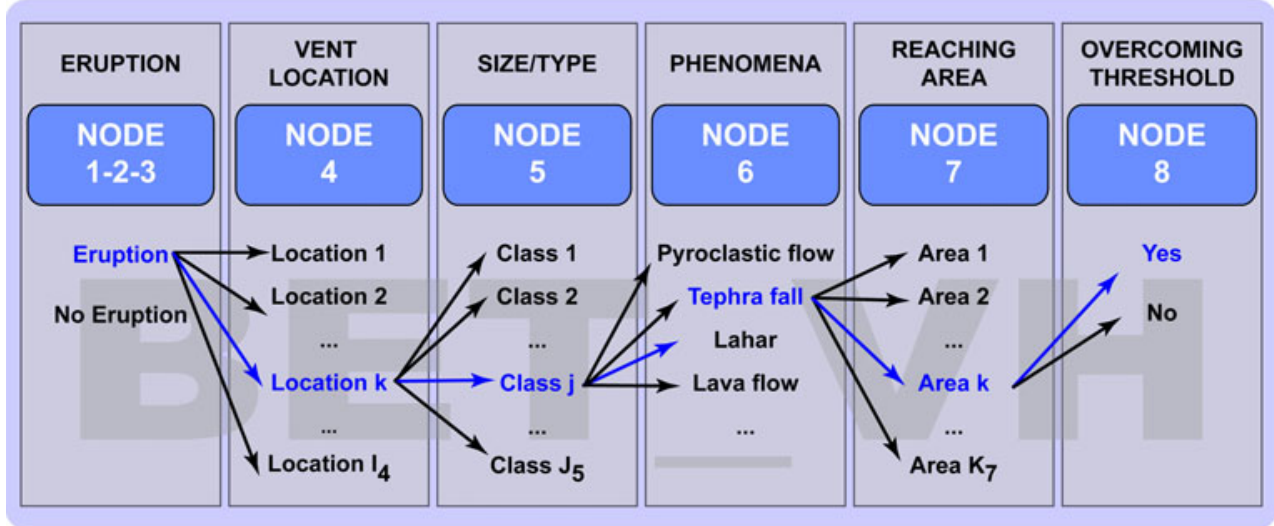

b

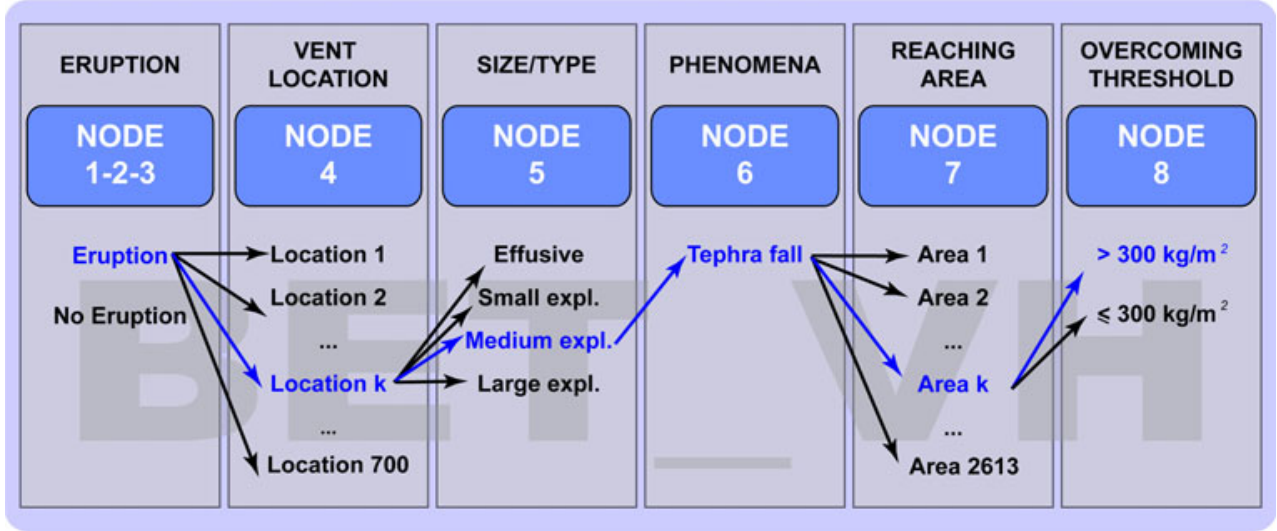

list of indexes and symbols used in this paper and in Marzocchi et al. (2010). Note that here and in Marzocchi et al. (2010), each index reported in Table 1 refers always to the same variable (e.g. $i$ refers always to vent positions etc.).

The prior distribution of the volcanic outcome (i.e. tephra fall in this application) at a generic $k$-th area produced by an eruption occurred at a generic $i$-th vent location and the generic $j$-th size class is completely defined by $\Theta_{7 ; i, j}^{(k)}$ and $\Lambda_{7 ; i, j}^{(k)}$, for node 7 , and $\Theta_{8 ; i, j}^{(k)}$ and $\Lambda_{8 ; i, j}^{(k)}$, for node 8 . Such values represent the average probability and the equivalent number of data (related to the variance), for nodes 7 and 8 , respectively

Table 1 Indexes for the BET_VH parameters (see Marzocchi et al. 2010)

\begin{tabular}{ll}
\hline & Description \\
\hline$i$ & $i$-th vent location (node 4) \\
$k$ & $j$-th size class (node 5) \\
$r$ & $k$-th area for the outcome (node 7) \\
$s$ & $r$-th run of the model \\
\hline
\end{tabular}

(Marzocchi et al. 2010). Such parameters can be estimated as:

$$
\begin{aligned}
& \left\{\Theta_{7 ; i, j}^{(k)}=\frac{\bar{\alpha}_{7 ; i, j}^{(k)}}{\bar{\alpha}_{7 ; i, j}^{(k)}+\bar{\beta}_{7 ; i, j}^{(k)}}\left\{\frac{\bar{\alpha}_{7 ; i, j}^{(k)}+\bar{\beta}_{7 ; i, j}^{(k)}}{\bar{\alpha}_{7 ; i, j}^{(k)}+\bar{\beta}_{7 ; i, j}^{(k)}+\Lambda_{m}}\right\}\right. \\
& +\frac{v_{7 ; i, j}^{(k)}}{N_{r}}\left\{\frac{\Lambda_{m}}{\bar{\alpha}_{7 ; i, j}^{(k)}+\bar{\beta}_{7 ; i, j}^{(k)}+\Lambda_{m}}\right\} \\
& \Lambda_{7 ; i, j}^{(k)}=\bar{\alpha}_{7 ; i, j}^{(k)}+\bar{\beta}_{7 ; i, j}^{(k)}-1+\Lambda_{m} \\
& \Theta_{8 ; i, j, k}^{(s)}=\frac{\bar{\alpha}_{8 ; i, j, k}^{(s)}}{\bar{\alpha}_{8 ; i, j, k}^{(s)}+\bar{\beta}_{8 ; i, j, k}^{(s)}}\left\{\frac{\bar{\alpha}_{8 ; i, j, k}^{(s)}+\bar{\beta}_{8 ; i, j, k}^{(s)}}{\bar{\alpha}_{8 ; i, j, k}^{(s)}+\bar{\beta}_{8 ; i, j, k}^{(s)}+\Lambda_{m}}\right\} \\
& +\frac{v_{8 ; i, j, k}^{(s)}}{v_{7 ; i, j}^{(k)}}\left\{\frac{\Lambda_{m}}{\bar{\alpha}_{8 ; i, j, k}^{(s)}+\bar{\beta}_{8 ; i, j, k}^{(s)}+\Lambda_{m}}\right\} \\
& \Lambda_{8 ; i, j, k}^{(s)}=\bar{\alpha}_{8 ; i, j, k}^{(s)}+\bar{\beta}_{8 ; i, j, k}^{(s)}-1+\Lambda_{m}
\end{aligned}
$$

The parameters contained into these equations (see Table 2) are of two types: the model-dependent 
Table 2 Symbols for the BET_VH parameters (see Marzocchi et al. 2010)

\begin{tabular}{|c|c|c|}
\hline & Symbol & Description \\
\hline \multirow[t]{5}{*}{ Probability distribution } & {$[\Phi]$} & Absolute probability \\
\hline & {$\left[\theta_{1-2-3}\right]$} & Absolute probability of eruption in the next $\tau$ \\
\hline & {$\left[\phi_{E S}\right]$} & $\begin{array}{l}\text { Conditional probability of the selected ES(s), } \\
\text { given an eruption }\end{array}$ \\
\hline & {$\left[\phi_{c}\right]$} & $\begin{array}{l}\text { Conditional probability at node } 7 \text {, given eruption } \\
\text { and ES(s) }\end{array}$ \\
\hline & {$\left[\phi_{d}\right]$} & $\begin{array}{l}\text { Conditional probability at node } 8 \text {, given eruption } \\
\text { and ES(s) }\end{array}$ \\
\hline \multirow[t]{9}{*}{ Parameters } & $I_{4}$ & Total number of vent location (node 4) \\
\hline & $J_{5}$ & Total number of size classes (node 5) \\
\hline & $K_{7}$ & Total number of areas classes (node 7) \\
\hline & $\Theta_{6 ; j}$ & Conditional average probability of prior model (node 6 ) \\
\hline & $\Lambda_{6 ; j}$ & Equivalent number of data of prior model (node 6) \\
\hline & $\Theta_{7 ; i, j}^{(k)}$ & $\begin{array}{l}\text { Conditional average probability of the prior model } \\
\text { (node } 7 \text { ) }\end{array}$ \\
\hline & $\Lambda_{7 ; i, j}^{(k)}$ & Equivalent number of data of prior model (node 7) \\
\hline & $\Theta_{8 ; i, j}^{(k)}$ & $\begin{array}{l}\text { Conditional average probability of the prior model } \\
\text { (node } 8 \text { ) }\end{array}$ \\
\hline & $\Lambda_{8 ; i, j}^{(k)}$ & Equivalent number of data of prior model (node 8 ) \\
\hline \multirow[t]{2}{*}{ Hyper-prior } & $\bar{\alpha}_{7 ; i, j}^{(k)}, \bar{\beta}_{7 ; i, j}^{(k)}$ & Hyper-prior parameters at node 7 \\
\hline & $\bar{\alpha}_{8 ; i, j, k}^{(s)}, \bar{\beta}_{8 ; i, j, k}^{(s)}$ & Hyper-prior parameters at node 8 \\
\hline \multirow[t]{7}{*}{ Theoretical model } & $\Lambda_{m}$ & Equivalent number of data relative to the model adopted \\
\hline & $N_{r}$ & N. of runs of the model \\
\hline & $\pi_{i, j, k, r}$ & Model output values \\
\hline & $v_{7 ; i, j}^{(k)}$ & N. of reaching episodes (node 7) out of the $N_{r}$ runs \\
\hline & $v_{8 ; i, j, k}^{(s)}$ & N. of overcoming episodes (node 8) out of the $N_{r}$ runs \\
\hline & $w_{7}$ & Normalized weight for runs at node 7 (Eq. 9) \\
\hline & $w_{8}$ & Normalized weight for runs at node 8 (Eq. 15) \\
\hline
\end{tabular}

parameters $\left(N_{r}, v_{7 ; i, j}^{(k)}, v_{8 ; i, j, k}^{(s)}\right.$ and $\left.\Lambda_{m}\right)$ and the hyperprior parameters $\left(\bar{\alpha}_{7 ; i, j}^{(k)}, \bar{\beta}_{7 ; i, j}^{(k)}, \bar{\alpha}_{8 ; i, j, k}^{(s)}\right.$ and $\left.\bar{\beta}_{8 ; i, j, k}^{(s)}\right)$.

\section{Hyper-prior parameters}

The hyper-prior parameters are relative to a sort of hyper-prior Beta distribution for the nodes 7 and 8 (see "Appendix"). The use of such a hyper-prior distribution has two main goals.

The first goal is to substitute the value of zero probability for the areas that have never hit by numerical simulations with a more realistic (low) probability. In this case, these values have to represent a first-order guess of the probability at nodes 7 and 8 , and they can be defined using empirical observations in other volcanoes (see, for instance, Tables 2 and 3 in Newhall and Hoblitt 2002), whenever such generalization is assumed to hold. This point is particularly important when the model(s) does not cover all the possible ESs combinations. In the simplest case, we can set $\bar{\alpha}_{7 ; i, j}^{(k)}=\bar{\beta}_{7 ; i, j}^{(k)}=\bar{\alpha}_{8 ; i, j, k}^{(s)}=$ $\bar{\beta}_{8 ; i, j, k}^{(s)}=1$, reproducing the least informative starting hypothesis (see discussion in Marzocchi et al. 2004, 2008, 2010).

The second and maybe most important goal of the hyper-prior parameters is that they allow different models, with different coverage, resolution and physical details, to be applied recursively. In practice, the parameters of the Beta distribution obtained from one model can be set as new hyper-prior parameters for the second model and so on (see "Appendix" for further details).

\section{Model-dependent parameters}

The model-dependent parameters are $N_{r}, v_{7 ; i, j}^{(k)}, v_{8 ; i, j, k}^{(s)}$ and $\Lambda_{m}$. The parameter $N_{r}$ is the number of runs of a model. The runs are produced to account for the statistical variability of the model input, given an eruption occurred in a specific vent location (node 4) and size class (node 5). For example, for tephra dispersion 
models, the runs account for the statistical variability of the winds distribution and eruptive conditions.

The parameter $v_{7 ; i, j}^{(k)}$ counts the number of times, out of the $N_{r}$ runs of the model, for which the $k$-th area is reached by the volcanic outcome, given an eruption of size class $j$ occurred at the vent location $i$; note that $v_{7 ; i, j}^{(k)}$ is the results of a counting over the model's results, and it may be equal to 0; thus, Eq. 2 may result undefined. In this case, the values of $\Theta_{8 ; i, j, k}^{(s)}$ and $\Lambda_{8 ; i, j, k}^{(s)}$ rely completely to the hyper-prior parameters (see "Appendix" for further details). In analogy, $v_{8 ; i, j, k}^{(s)}$ counts the number of times, out of the $v_{7 ; i, j}^{(k)}$, for which the threshold $s$ of the volcanic outcome is exceeded (e.g. in terms of loading, dynamic pressure etc.) in the $k$-th area, given an eruption of size class occurred at the vent location $i$.

The only model-dependent parameter that is not set by the runs is $\Lambda_{m}$, i.e. the equivalent number of data to be assigned to the model. $\Lambda_{m}$ must be set depending on the (subjective) reliability that the researcher gives to the modeling procedure adopted, i.e. not only to the model itself but also the underlying assumptions and the capability of the $N_{r}$ realizations to successfully describe the whole physical system (e.g. for tephra fall, wind variability, emission rates variability during the eruption, DEM models etc.). The choice of $\Lambda_{m}$ is subjective, but it is unavoidable. In practice, $\Lambda_{m}$ controls the decrease of the variance of the Beta distribution, i.e. the uncertainty on the estimation, due to the model results (see "Appendix" and Marzocchi et al. (2010) for further details).

\section{Application to tephra fallout at the Campi Flegrei caldera}

In this application, we focus on the tephra fallout hazard assessment for CFc. In particular, we analyze the effects of combining the output of different ESs compared to the same hazard calculated for just one single ES, where the uncertainty in the vent location and in the size class of the eruption are neglected. The event tree used for this application is reported in Fig. 1b. In the following, we describe the setting for each node and the tephra fallout model used, providing a complete tutorial for BET_VH applications (Marzocchi et al. 2010) and a description of the scheme to integrate output of different models (previous paragraph and "Appendix"). The results of the application to the CFc will be discussed in the sections below.
BET_VH: nodes 1 to 6

All the hazard maps shown in the followings will be conditioned to the occurrence of an eruption. For this reason, we do not focus our attention on the probability estimation at nodes 1-2-3 (probability of eruption in a time window $\tau$ ). We remark that such an estimation would translate all the conditioned probability maps shown in this paper into absolute probability maps (see Marzocchi et al. 2010).

For node 4, we consider the probability map of vent opening proposed by Selva et al. (2007); this map accounts for geostructural knowledge of the caldera as well as past data observed for the last $5 \mathrm{kyr}$. The $\mathrm{CFc}$ is divided into 700 possible square vent locations $\left(I_{4}=700\right)$ with size of $500 \mathrm{~m}$. The probability map of best guess values is reported in Fig. 2a.

For node 5, according to the results of the recent Italian project V3-2 Campi Flegrei (ended in July 2007, DPC/INGV PROJECT V3 2004-2006), we classify $\mathrm{CFc}$ eruptions in four representative size classes $\left(J_{4}=4\right)$, regardless of the vent location: effusive eruptions, small, medium and large explosive eruptions. Such grouping is based on the analysis of several parameters (in particular volume and magnitude that, for $\mathrm{CFc}$, show a good agreement); an exhaustive description of such size classes can be found in Orsi et al. (2009) and references therein. Basically, the prior distribution of the size class probability is set through a power-law distribution for explosive classes (classes 2 to 4), observed in the size distribution of worldwide eruptions, plus a 0.05 probability for effusive eruptions (size class 1); this prior distribution is then combined with past data of the last $5 \mathrm{kyr}$. All of this information contributes to form the posterior distribution for node 5 , giving best guess probabilities as reported in Fig. $2 \mathrm{~b}$.

For node 6, we assume that all eruptions of size classes 2 or larger certainly produce tephra; eruptions of size class 1 do not produce tephra (Simkin and Siebert 1994; Newhall and Hoblitt 2002). This choice is certainly a simplification and it implies the assumption that the effect of tephra dispersal from eruption of size class 1 is negligible respect to the ones from the other classes. This is justified by the fact that past effusive eruptions generated only small-volume lava domes and lava flows, while explosive eruptions generated particles fallout (e.g. Orsi et al. 2009 and references therein). In terms of BET_VH symbols, we have $\Theta_{6 ; 1}=0, \Theta_{6 ; 2}=$ $\Theta_{6 ; 3}=\Theta_{6 ; 4}=1$. These values of $\Theta(0$ or 1 , states of certainty) imply that in the Beta distributions, either $\alpha$ or $\beta$ will be equal to 0 . In this case, the equivalent number of 
Fig. 2 Average (best guess) conditional probability of (a) vent opening, i.e. node 4 from Selva et al. (2007), and (b) size class of eruption, i.e. node 5, from Orsi et al. (2009)
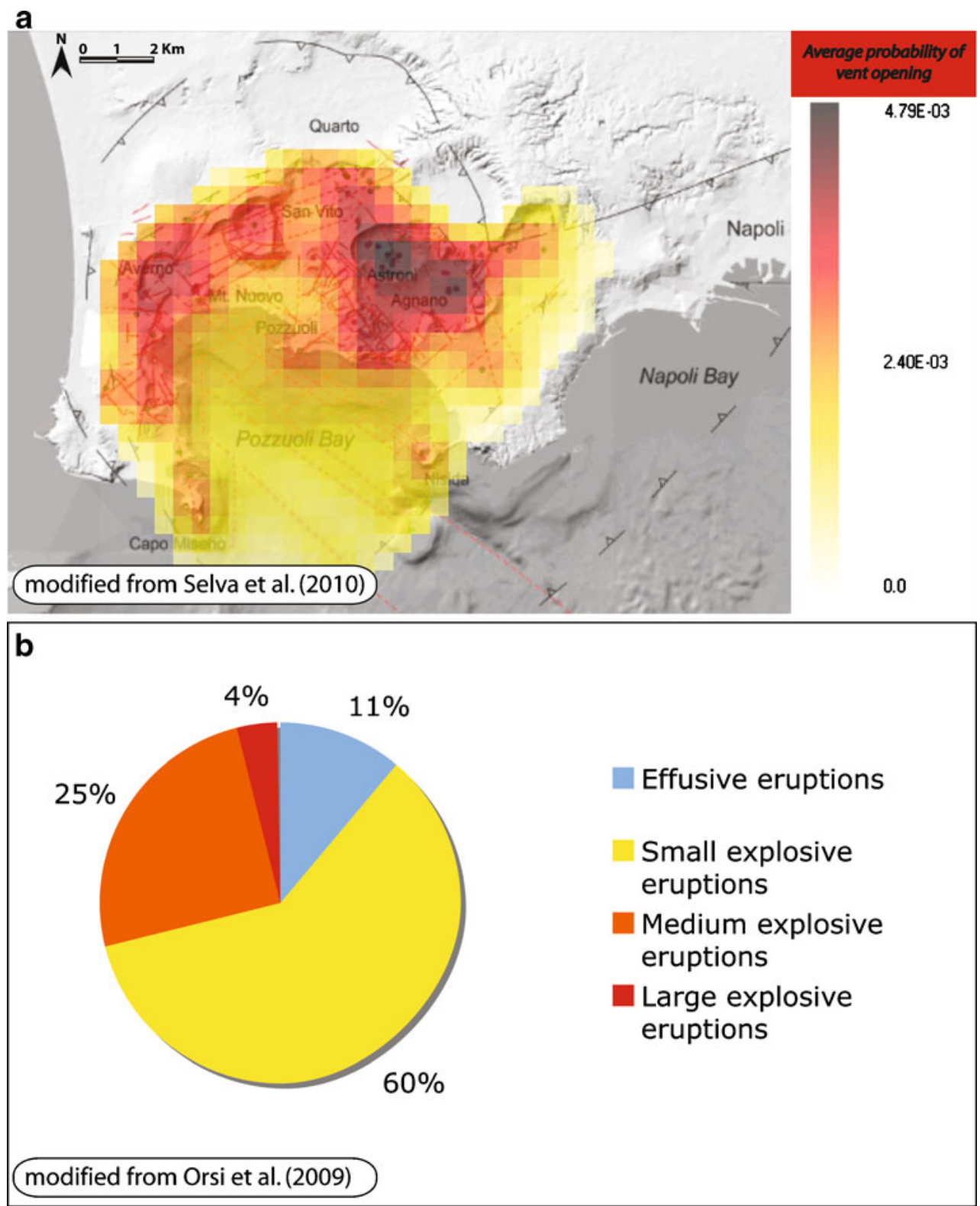

data $\left(\Lambda_{6 ; j}\right)$ is not relevant, and the Beta distribution will be a Dirac's delta centered in 0 and 1, respectively, i.e. prior probabilities are expressed in terms of certainties and thus the probability distributions will have zero variance (see, e.g. Marzocchi et al. 2004; Gelman et al. 1995).

\section{Tephra fallout model}

Dispersion and sedimentation of volcanic particles released from a sustained eruption column in the medium and distal areas can be fairly well determined from wind transport, turbulent diffusion and settling by gravity. For relatively small computational domains (i.e. $<100 \mathrm{~km}$ ) and short durations (i.e. $<$ few days), wind field can be assumed to be constant and horizontally uniform. Moreover, vertical diffusion coefficient and vertical wind component can be reasonably neglected. Under these assumptions, the mass conservation equation for each class of particles with a given settling velocity can be solved by using a semi-analytical solution as described in Macedonio et al. (2005) and Pfeiffer et al. (2005).

Here, the goal is to model all possible kinds of fallout deposit produced by sustained columns at $\mathrm{CFc}$, i.e. to cover all the possible combinations of vent position and size class (ESs). As a first approximation, we choose to neglect orography effects. This is a reasonable assumption since in the case of $\mathrm{CFc}$, topographic differences are much smaller than the eruption columns 
we consider in this study. Therefore, we model the tephra dispersion for all the three size classes producing tephra, but using only one single vent location; then, we translate the virtual vent to each of all the possible 700 vent locations.

In order to set the emission rate and volume of the simulated eruptions, we select single values to each one of the size classes, simulating one reference eruption size for each class. We select the Agnano Monte Spina, the Astroni 6 and the Averno 2 eruptions as representative of the high-, medium- and low-magnitude eruptions, following Orsi et al. (2004), Costa et al. (2009) and Orsi et al. (2009). The input parameters of these three reference eruptions can be found in Table 3. In order to account for possible wind distribution variations, for each size class, we compute the solution relative to 13,149 different wind profiles for the Campanian region, i.e. 36 years of daily wind profiles, obtained from National Oceanic and Atmospheric Administration (NOAA) reanalysis (Costa et al. 2009).

The approximation of using a fixed emission rate for each size class is comparable to deterministic approaches (e.g. Barberi et al. 1990; Macedonio et al. 2008). It may be argued that this approximation may introduce a bias into the final hazard assessment because an eruption of a generic size class is usually characterized by a range of emission rates not just by a single value (Cioni et al. 2003). Nonetheless, the variability of the emission rate among different size classes is much larger than the variability of the emission rate for one specific size class. Therefore, a hazard assessment that accounts for the variability of different ESs (as we will show later) may be only slightly affected by the inclusion of variability of emission rates within each size class.
Finally, it is worth noting that the assumption of a constant wind (we consider only daily wind variations) may be not general for small scale eruptions (sometimes characterized by a prolonged activity over periods of days to weeks, during which low-level winds can be largely variable). However, in the case of the eruption chosen as reference for the low-magnitude eruption, i.e. Averno 2, the proximal deposit shows a uniaxial distribution (Costa et al. 2009) that justifies this assumption.

\section{BET_VH: nodes 7 and 8}

To set the prior probabilities at nodes 7 and 8 , we follow the scheme presented above and in "Appendix". First, we define the potentially impacted region as a rectangle of $80 \times 100 \mathrm{~km}$. Then, we divide it into 2,613 areas with size increasing with the distance from the caldera. In each of these areas, the probability is assumed to be homogeneous. The distance between the centers of adjacent areas ranges from a minimum of $1 \mathrm{~km}$ (within the caldera) to a maximum of $4 \mathrm{~km}$ (at the limit of the rectangular region); Fig. 3 shows a map representing the central points of each cell. As regards the threshold for tephra loading at node 8 , we select $s=300 \mathrm{~kg} \mathrm{~m}^{-2}$ according to Costa et al. (2009) and references therein.

To limit the CPU time for the evaluation of the BET_VH parameters, we randomly selected 1,000 runs of the model $\left(N_{r}=1,000\right)$ among the 13,149 different wind profiles. This choice does not affect the results since this will only limit (approximately to 0.01 ) the precision of the estimate. In principle, we can even select only runs relative to a given season for which the wind variability is smaller, obtaining the probability
Table 3 Input parameters used for the different size classes, from Table 1 in Costa et al. (2009)

\begin{tabular}{|c|c|c|c|}
\hline Model parameters & Large explosive & Medium explosive & Small explosive \\
\hline Total mass & $5.2 \times 10^{11} \mathrm{~kg}$ & $1.2 \times 10^{11} \mathrm{~kg}$ & $2.3 \times 10^{10} \mathrm{~kg}$ \\
\hline Column height & $26 \mathrm{~km}$ & $12 \mathrm{~km}$ & $7 \mathrm{~km}$ \\
\hline Column shape coefficients & $4 / 1$ & $4 / 1$ & $3 / 1$ \\
\hline Number of $V_{\text {sett }}$ classes & 6 & 6 & 6 \\
\hline Bulk settling velocity & $0.5 \mathrm{~m} / \mathrm{s}(18)$ & $0.5 \mathrm{~m} / \mathrm{s}(12)$ & $0.5 \mathrm{~m} / \mathrm{s}(10)$ \\
\hline \multirow{5}{*}{ distribution $V_{\text {sett }}($ wt.\%) } & $2.5 \mathrm{~m} / \mathrm{s}(52)$ & $2.5 \mathrm{~m} / \mathrm{s}(28)$ & $2.5 \mathrm{~m} / \mathrm{s}(16)$ \\
\hline & $4.5 \mathrm{~m} / \mathrm{s}(18)$ & $4.5 \mathrm{~m} / \mathrm{s}(36)$ & $4.5 \mathrm{~m} / \mathrm{s}(29)$ \\
\hline & $6.5 \mathrm{~m} / \mathrm{s}(6)$ & $6.5 \mathrm{~m} / \mathrm{s}(17)$ & $6.5 \mathrm{~m} / \mathrm{s}(26)$ \\
\hline & 8.5 m/s (3) & $8.5 \mathrm{~m} / \mathrm{s}(5)$ & $8.5 \mathrm{~m} / \mathrm{s}(12)$ \\
\hline & $10.5 \mathrm{~m} / \mathrm{s}(3)$ & $10.5 \mathrm{~m} / \mathrm{s}(2)$ & $10.5 \mathrm{~m} / \mathrm{s}(7)$ \\
\hline Diffusion coefficient $K$ & $5,000 \mathrm{~m}^{2} / \mathrm{s}$ & $5,000 \mathrm{~m}^{2} / \mathrm{s}$ & $1,000 \mathrm{~m}^{2} / \mathrm{s}$ \\
\hline Mass eruption rate & $\lesssim 10^{8} \mathrm{~kg} / \mathrm{s}$ & $\lesssim 10^{7} \mathrm{~kg} / \mathrm{s}$ & $\lesssim 10^{6} \mathrm{~kg} / \mathrm{s}$ \\
\hline $\begin{array}{l}\text { Daily wind profiles } \\
\text { (period 1968-2003) }\end{array}$ & $\begin{array}{l}\text { NOAA } \\
\text { (latitude } 40^{\circ} ; \\
\text { longitude } 15^{\circ} \text { ) }\end{array}$ & $\begin{array}{l}\text { NOAA } \\
\text { (latitude } 40^{\circ} ; \\
\text { longitude } 15^{\circ} \text { ) }\end{array}$ & $\begin{array}{l}\text { NOAA } \\
\text { (latitude } 40^{\circ} ; \\
\text { longitude } 15^{\circ} \text { ) }\end{array}$ \\
\hline
\end{tabular}


Fig. 3 Grid point for the probability maps at nodes 7 and 8 . The step of the grid becomes larger moving away from the possible sources of eruptions; see text for more details. Adapted from Google Earth (2010 Europa

Technologies; 2010 Tele Atlas)

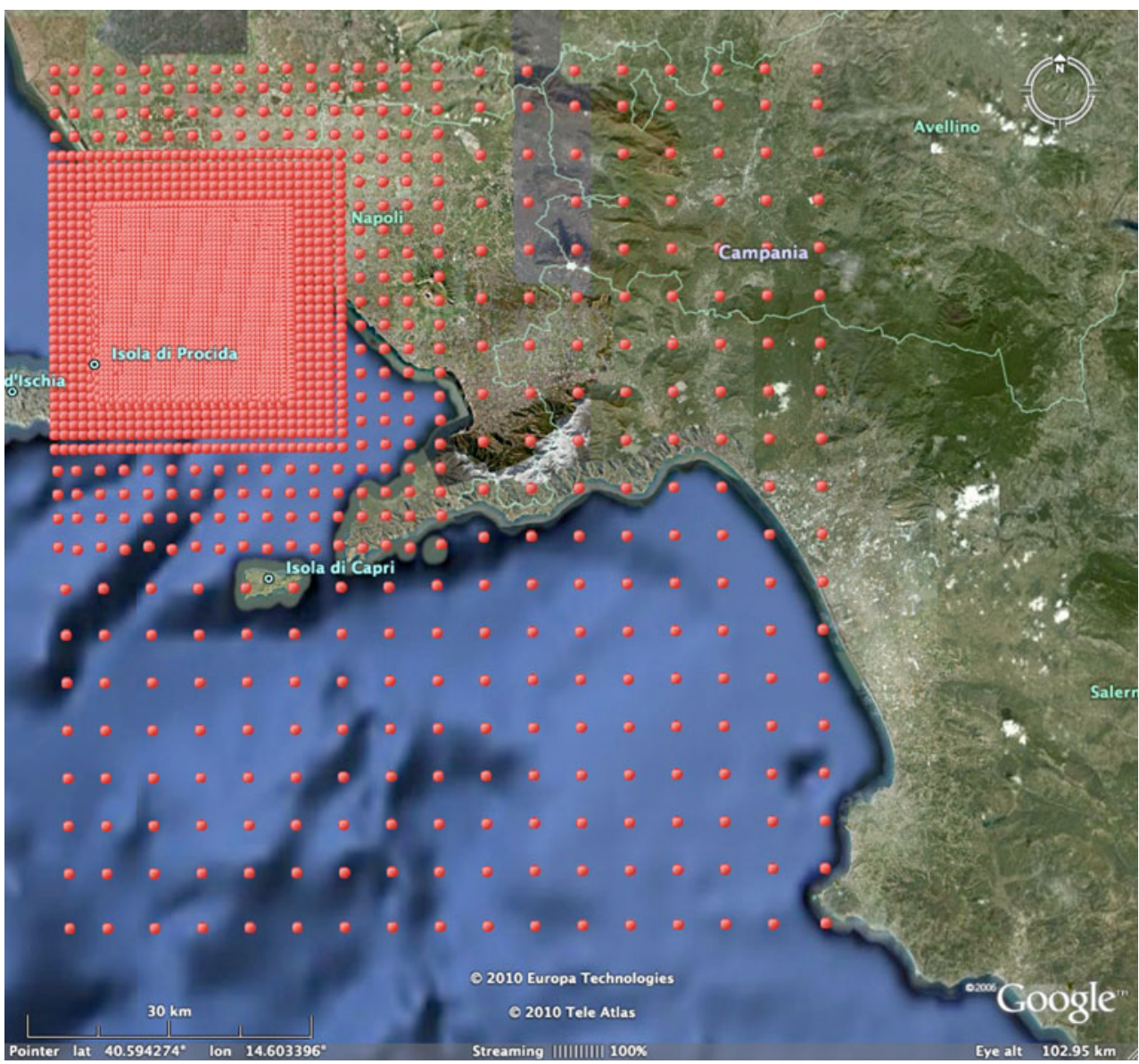

for an event occurring in spring, summer, autumn and winter, respectively.

Finally, we set the equivalent number of data of the model $\Lambda_{m}=10$ and $\bar{\alpha}_{7 ; i, j}^{(k)}=\bar{\beta}_{7 ; i, j}^{(k)}=\bar{\alpha}_{8 ; i, j, k}^{(s)}=\bar{\beta}_{8 ; i, j, k}^{(s)}=1$. The choice of taking a uniform distribution for the hyper-prior is not critical since the fallout model results are assigned a much larger weight $\left(\Lambda_{m}=10\right)$, with the model covering all the considered ESs, i.e. all size classes and vent locations.

With the choices described above, we have the following input parameters for node 7 :

$\Theta_{7 ; i, j}^{(k)}=\frac{1}{2}\left\{\frac{2}{12}\right\}+\frac{v_{7 ; i, j}^{(k)}}{1,000}\left\{\frac{10}{12}\right\}$

$\Lambda_{7 ; i, j}^{(k)}=11$

where $i=1,2, \ldots, 700$ indicates the vent location, $j=$ $2,3,4$ indicates the size class, $k$ refers to the $k$-th area and $v_{7 ; i, j}^{(k)}$ is the number of times that tephra reaches the $k$-th area in the 1,000 random realizations of the model (see Table 2).

Similarly, at node 8 , we have:

$\Theta_{8 ; i, j, k}^{(s)}= \begin{cases}\frac{1}{2}\left\{\frac{2}{12}\right\}+\frac{v_{8 ; i, j, k}^{(s)}}{v_{7 ; i, j}^{(k)}}\left\{\frac{10}{12}\right\} & \text { if } v_{7 ; i, j}^{(k)}>0 \\ \frac{1}{2} & \text { if } v_{7 ; i, j}^{(k)}=0\end{cases}$

$\Lambda_{8 ; i, j, k}^{(s)}= \begin{cases}11 & \text { if } v_{7 ; i, j}^{(k)}>0 \\ 1 & \text { if } v_{7 ; i, j}^{(k)}=0\end{cases}$

where $v_{8: i, j, k}^{(s)}$ is the number of times that the selected threshold of $300 \mathrm{~kg} \mathrm{~m}^{-2}$ is overcome in the $k$-th area in the 1,000 random realizations of the model (see Table 2).

\section{Results}

To analyze the effects of the combined ESs approach, we present the results relative to a selection of single 
and combined ESs. As single ESs, we select the following settings:

1. S1: probability of an eruption similar to the Averno 2 event. For this ES, we select vent location 480 at node 4 and size class 2 at node 5 .

2. S2: probability of an eruption similar to the Astroni 6 (prototype of medium explosive eruptions at $\mathrm{CFc}$ ). For this ES, we select vent location 521 at node 4 and size class 3 at node 5 .

According to the results reported by Orsi et al. (2009), S1 reflects the most likely size class of eruptions (small explosive), while S2 represents the medium explosive eruption class, often adopted for civil protection application (e.g. for Vesuvio, Barberi et al. 1995, 2008; DPC 1995).

We also consider the following ESs combinations:

1. COMBO1: probability of an eruption of any size class in the most probable vent location, i.e. within the Agnano crater (see Fig. 2). For this case, we select vent location 520 at node 4 and all size classes at node 5 .

2. COMBO2: probabilities of an eruption of any size class from all possible vent locations. For this case, we select all vent locations at node 4 and all size classes at node 5; each vent and size class is weighted by its probability of occurrence.

In summary, both COMBO1 and COMBO2 are a weighted average of ESs, where each ES is weighted by its own probability of occurrence (see Marzocchi et al. 2010, Eqs. 20 and 21). Both COMBO1 and $\mathrm{COMBO} 2$ account for all combinations of size classes. Only COMBO2 also includes the variability of all vent locations.

The results for S1, S2, COMBO1 and $\mathrm{COMBO} 2$ cases are reported in Figs. 4, 5, 6 and 7, respectively; we plot the conditional best guess (average) probability maps for node 7 (upper panel, probability that tephra will reach a specific area) and for node 8 (lower panel, probability that tephra will overcome a loading of $300 \mathrm{~kg} \mathrm{~m}^{-2}$ in each area). Referring to the symbols in Marzocchi et al. (2010, Eqs. 20 and 21), we plot the average of $\left[\phi_{c}\right]$ and $\left[\phi_{d}\right]$ for areas $k=1, \ldots, 2613$. Note that the maps relative to the node 8 are zoomed. All these probabilities are conditional to the occurrence of an eruption belonging to the selected single or combined ESs. The absolute probability $[\Phi]$ in the time window $\tau$ is obtained multiplying these probabilities by the conditional probability of the selected single or combined ESs $\left(\left[\phi_{E S}\right]\right)$ and the probability to have an eruption in the time frame $\tau\left(\left[\theta_{1-2-3}\right]\right.$, independent from the ES), that is

$[\Phi]=\left\{\begin{array}{l}{\left[\theta_{1-2-3}\right]\left[\left[\phi_{E S}\right]\left[\phi_{c}\right] \text { for node } 7\right.} \\ {\left[\theta_{1-2-3}\right]\left[\left[\phi_{E S}\right]\left[\phi_{d}\right] \text { for node } 8\right.}\end{array}\right.$

Notably, the conditional probability of occurrence of single or combined ESs, i.e. $\left[\phi_{E S}\right]$, strongly varies across the possible ESs. We may interpret such probabilities as the relative weight of each output probability map (at nodes 7 and 8, as in Figs. 4-7). In other words, if $\left[\phi_{E S}\right]$ is relatively small, there is a relatively high probability that next eruption will occur with a different $\mathrm{ES}$, i.e. the map will not be representative. On the contrary, if the probability of occurrence $\left[\phi_{E S}\right]$ is close to 1 , it means that the output map is based on almost the totality of the possible eruptive settings (in terms of size class and vent location), i.e. the characteristics of the next eruption will be represented well by the probability values shown in the map. Therefore, $\left[\phi_{E S}\right]$ symbolizes the degree of representativeness of the map with respect to all possible ESs. As regards the selected ESs, S1, S2, COMBO1 and COMBO2, the average of $\left[\phi_{E S}\right]$ is $2.24 \times 10^{-3}, 1.19 \times 10^{-3}, 4.79 \times 10^{-3}$ and 1.0 , respectively. Note that the representativeness of COMBO1 is several times greater than the single ESs S1 and S2. On the other hand, COMBO2 has a representativeness three orders of magnitude greater than all the other cases (S1, S2 and COMBO1).

The results for S1 and S2 were discussed by Costa et al. (2009). Here, we highlight the comparison between results for single ESs (S1 and S2) and those obtained considering a combination of ESs (COMBO1 and $\mathrm{COMBO} 2$ ). As it appears clear from the figures, the maps relative to $\mathrm{COMBO} 1$ and $\mathrm{COMBO} 2$ plotted in Figs. 6 and 7 are significantly different from those obtained for single ESs (Figs. 4 and 5). Figure 6, relative to COMBO1, shows the effect of the uncertainty on the eruption size. Even though the most likely size class is the smallest explosive one, the probability map for COMBO1 (at both nodes 7 and 8 ) is more spread than for S1 (Fig. 4). This is due to the fact that COMBO1 considers events of greater size classes; therefore, more distant areas can be reached by tephra. Notably, the results for COMBO1, in terms of most dangerous areas, are between the ones obtained for S1 and S2. This may have an important impact in PVHA for central volcanoes, where the uncertainty on vent location is relatively less important; in fact, the extension of the most likely damaged areas (node 8) in COMBO1 is smaller than the extension of the most likely damaged areas for $\mathrm{S} 2$, where only and event of size class 3 is modeled. In 
Fig. 4 Probability maps for node 7 (a) and node 8 (b) for S1 (Averno 2). Further details can be found in the text.

Adapted from Google Earth (2010 Europa Technologies; 2010 Tele Atlas)
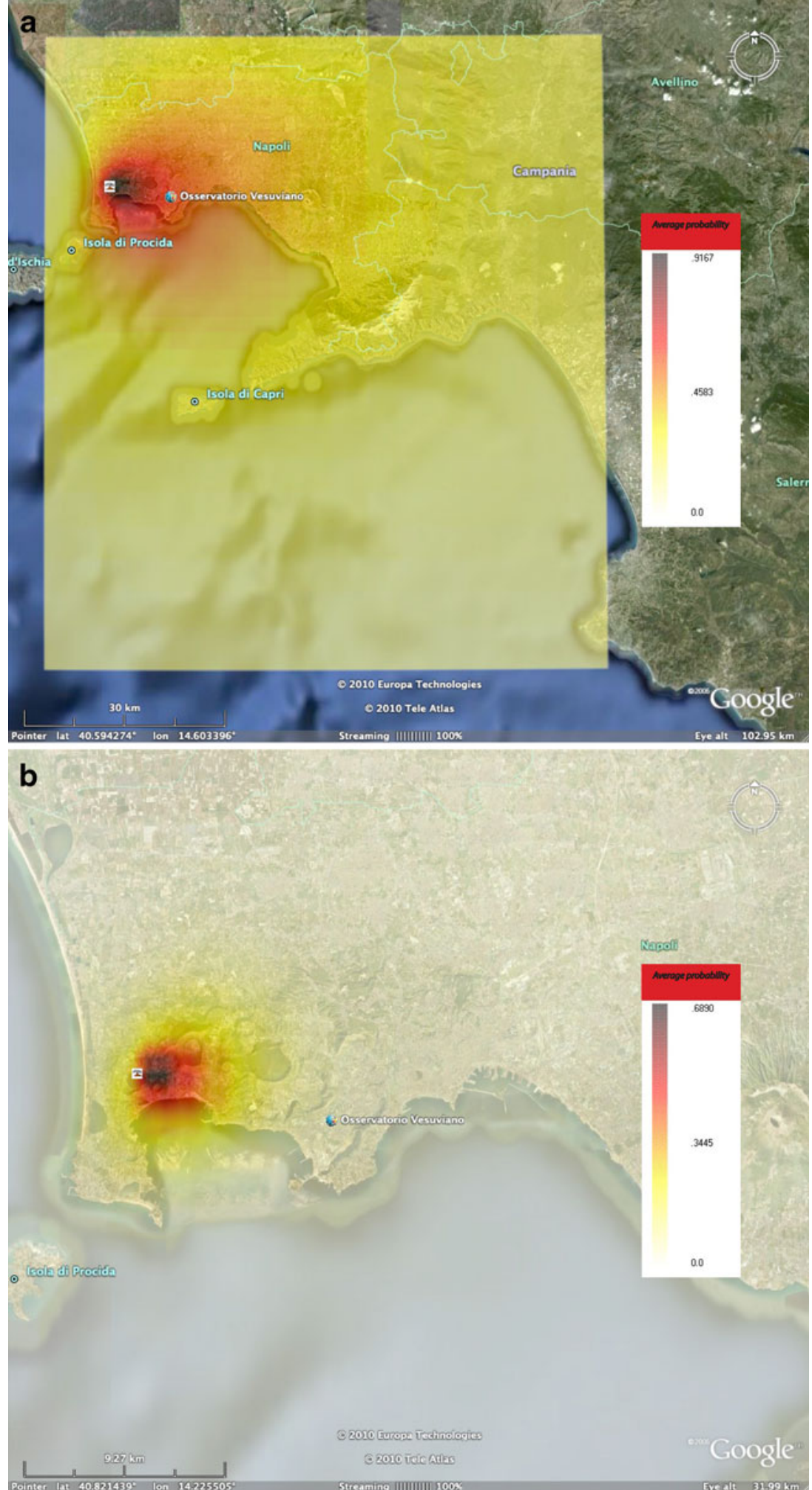

other words, if the latter is taken as reference event, we show that the hazard map derived by this event may represent an overestimation of the real hazard.

In Fig. 7, the effect of the inclusion of vent location uncertainty on the probability estimation for nodes 7 and 8 is shown. At CFc, as well as in all large calderas with many possible eruptive centers, such spatial uncertainty is one of the greatest sources of uncertainty. In fact, the changes in the probability maps are striking. In particular, the spatial uncertainty strongly increases 
Fig. 5 Probability maps for node 7 (a) and node 8 (b) for S2 (Astroni 6). Further details can be found in the text.

Adapted from Google Earth (2010 Europa Technologies; 2010 Tele Atlas)
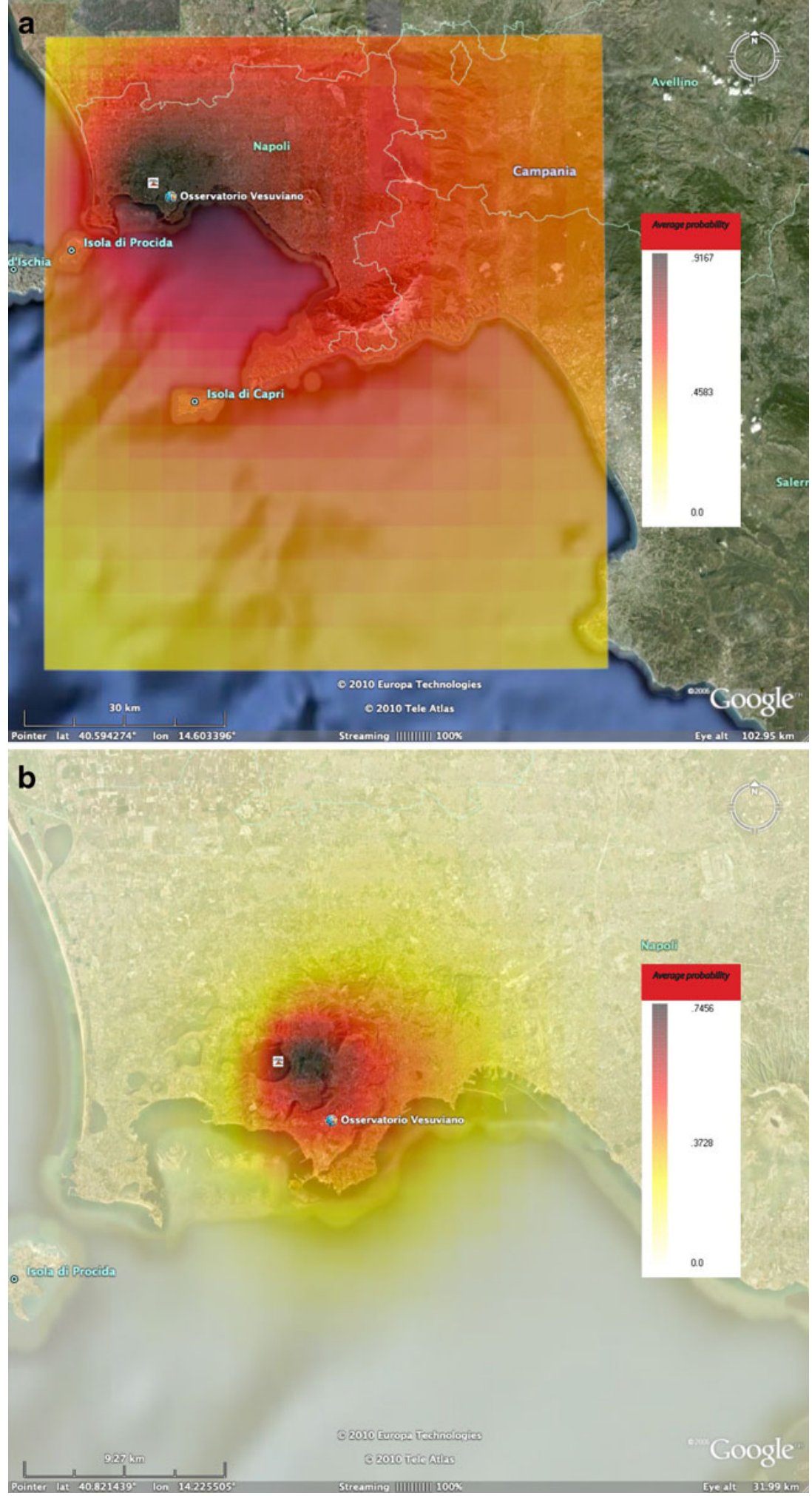

the spreading of the probability distribution since all areas inside the caldera find themselves close to one possible vent. A second aspect to be considered is that the maximum value of the conditional probability is lower than the maximum values in all previous cases (note the color scales in the figures). This is clearly an effect of the uncertainty in the vent location since the probability of high tephra loading close to a vent is 
Fig. 6 Probability maps for node 7 (a) and node 8 (b) for COMBO1 (most likely vent and all size classes). Further details can be found in the text. Adapted from Google Earth (2010 Europa

Technologies; 2010 Tele Atlas)
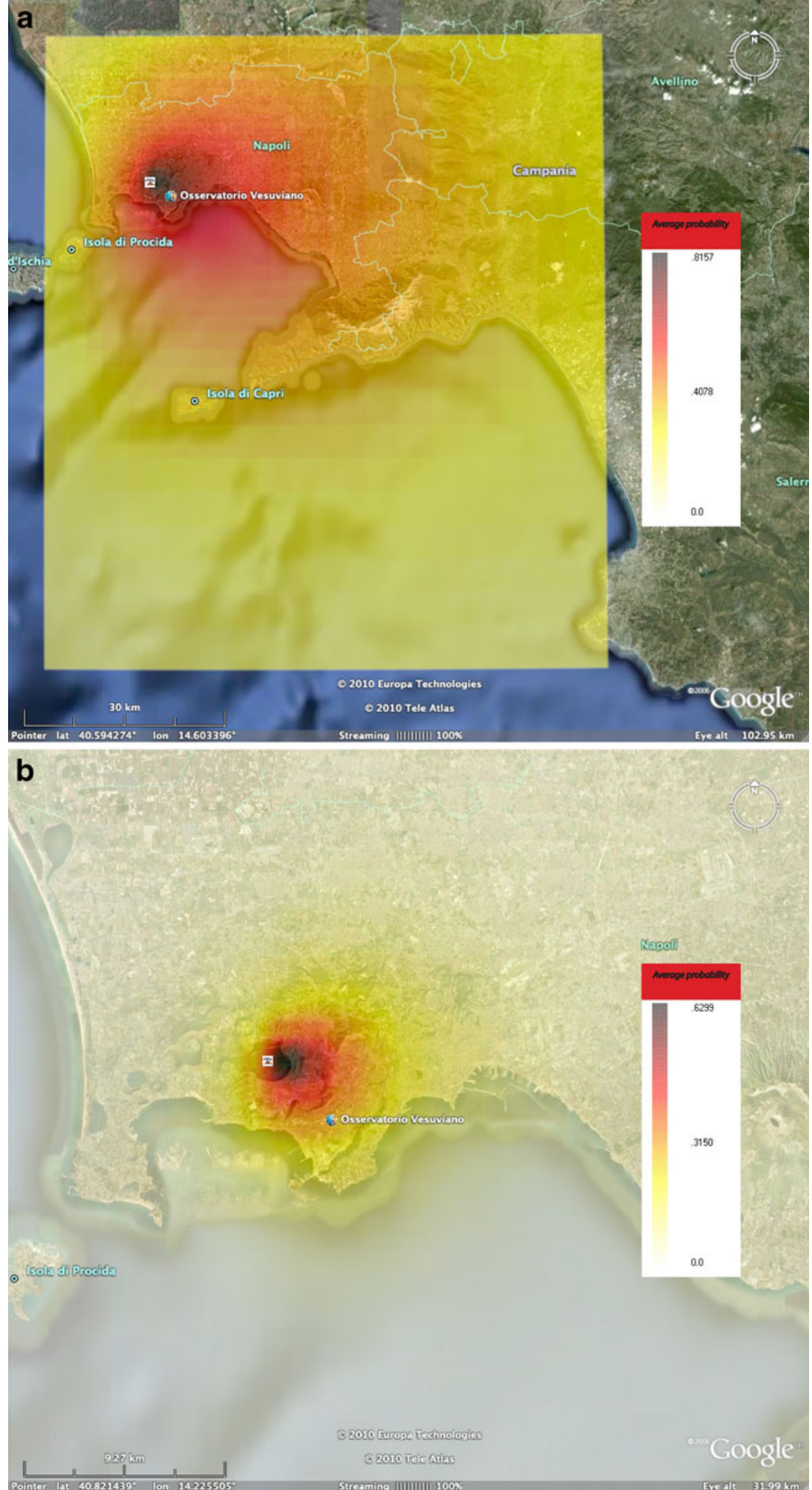

high, but the position of the vent is not certain as in the other cases (S1, S2 and COMBO1). That said, it is noticeable that the peak value of the absolute probability for $\mathrm{COMBO} 2$ is the largest since the relative $\left[\phi_{E S}\right]$ for $\mathrm{COMBO} 2$ is the highest compared to $\mathrm{S} 1, \mathrm{~S} 2$ and COMBO1 (see Eq. 7).

A third interesting feature to be noted is that the central-eastern part of the caldera has a non-negligible 
Fig. 7 Probability maps for node 7 (a) and node 8 (b) for COMBO2 (all vents and all size classes). Further details can be found in the text.

Adapted from Google Earth (2010 Europa Technologies; 2010 Tele Atlas)
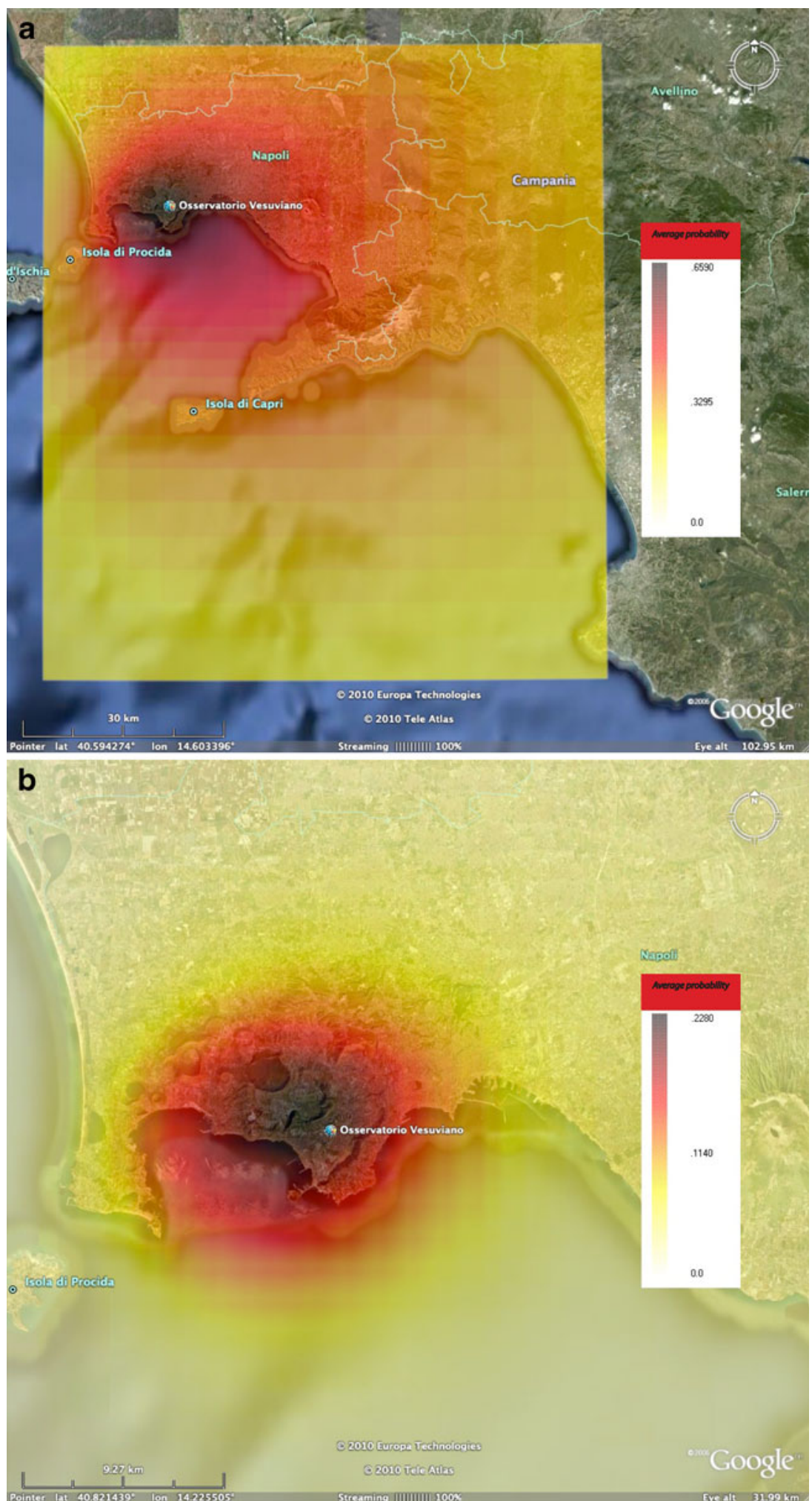

probability both at nodes 7 and 8; this is a stable and common feature that only partially depends on the vent opening probability distribution used (node 4; Fig. 1a). In fact, this feature mostly depends on the fact that this area is downwind with respect to the vast majority of vents, thus a stacking effect is present. The global result of the spatial uncertainty is that the probability of reaching and overcoming episodes has an almost 
uniform and relatively high (about 0.6 and 0.2 , respectively) value within the whole caldera, in particular in its central-eastern part.

\section{Discussion and final remarks}

The main goal of this paper is twofold: to explore the effects of including intrinsic uncertainties onto PVHA and to describe a realistic tutorial example for the use of the BET_VH code (described in the companion paper, Marzocchi et al. 2010) that can be easily extrapolated to many other applications. These goals are achieved through the application of the BET_VH model to the long-term tephra fall hazard for CFc.

The analysis reported here clearly shows that the inclusion of some uncertainties usually neglected in PVHA has a major impact on the final assessment. In particular, the use of combined ESs (COMBO 1 and COMBO 2) give results significantly different from single ESs (S1 and S2). The combination of all ESs generally gives smaller values of conditional probability and more spread distributions over the region around the volcano. As practical consequence of this, the most likely impacted area for the next eruption obtained combining different ESs is much larger than the one estimated by using a single ES, leading to a greater uncertainty about the most dangerous areas. This result suggests that the common practice in PVHA to use single "reference" events, such as the "maximum expected event", the "most likely event", or the "worse expected event", may lead to significant biases, in particular for calderas. Single ESs are not able to capture the wide variability of the hazard, and the combination of all possible ESs becomes essential to achieve an unbiased PVHA. Specifically, in this application, we note that the uncertainty in vent location plays a major role, and it must be included to get a reliable PVHA. This is a basic point because a reliable PVHA must be accurate (i.e. absence of significant biases) because a biased estimation would be useless in practice; on the other hand, it may have a low precision (i.e. a large uncertainty) that would reflect our scarce knowledge of some physical processes involved (Marzocchi et al. 2004, 2008).

Noteworthy, we do not attempt to obtain the "best" PVHA for $\mathrm{CF}$ c because this would require the inclusion of more models and field data. Anyway, we argue that this attempt, including different ESs, may represent a drastic reduction of bias into the long-term tephra fall hazard assessment for $\mathrm{CFc}$.

Finally, with this application, we have shown how BET_VH model may manage the output of different models, even models that describe just a single sce- nario, producing a prior probability distribution that averages them. This strategy has several similarities to the ensemble forecasting used in climate and weather forecasting (e.g. Krishnamurti et al. 2000), and it may be potentially used for any kind of volcanoes.

Acknowledgement A.C. acknowledges fundings from the Italian Department of Civil Protection (Research Project SPeeD).

Open Access This article is distributed under the terms of the Creative Commons Attribution Noncommercial License which permits any noncommercial use, distribution, and reproduction in any medium, provided the original author(s) and source are credited.

\section{Appendix: From simulation outputs to the prior distribution parameters at nodes 7 and 8}

In this appendix, we develop a possible scheme to convert the output of one or more theoretical models into prior probability distributions of BET_VH. This procedure is meant to be general and applicable to all possible volcanic phenomena (e.g. tephra fallout, pyroclastic flows, lahars, lava flows etc.), as well as to any possible theoretical model. In particular, here we present a formulation that directly links the simulation output results of a model to the distribution parameters at nodes 7 and 8 . Since the procedure may be applied recursively, such a formulation can be extended to more than one model.

In the companion paper (Marzocchi et al. 2010), we argue that a single run of a model, regardless of its intrinsic reliability, cannot be used to make a reliable forecasting, mostly due to the variability of the eruptive source and/or of the initial/boundary conditions. For instance, for tephra fall, even though the good knowledge of the atmospheric tephra transport leads to the availability of many reliable models (e.g. Bonadonna and Costa 2010), the modeling results strongly depend on wind distribution, column height, eruption intensity etc. In practice, we need to simulate a large number of different conditions (e.g. Costa et al. 2009). This set has to mimic the whole range of possible initial/boundary conditions, each one weighted with its own probability of occurrence. Based on this set of runs, we can evaluate hazard maps relative to single ESs (e.g. Bonadonna et al. 2002). Here, we describe how such a set of runs (together with the degree of reliability of the model itself) may be used to define the input parameters for the prior distribution at nodes 7 and 8 in BET_VH (see Marzocchi et al. 2010).

We define $N_{r}$ as the number of runs of a model that are produced to account for the statistical variability 
of the model input, given an eruption occurred in a specific vent location (node 4) and size class (node 5). As in Marzocchi et al. (2010), with the expression "size class", we mean either a specific size (e.g. V.E.I.), or type (e.g. effusive), or group of sizes (e.g. V.E.I. $\geq$ 3 ). We call $K_{7}$ the number of areas (either regularly spaced or not) in which the region around the volcano has been divided in order to model the outcome. With the symbol $\pi_{i, j, k, r}$, we indicate the output parameter of interest relative to the phenomenon (e.g. the loading for tephra fall or the dynamic pressure for pyroclastic density currents) in the $k$-th area for the $r$-th run of the model simulating an eruption occurring in vent location $i$ and of size class $j$. We call reaching episode in a given area every run of a model in which the phenomenon reaches the area. In analogy, we call overcoming episode every time a certain threshold $s$ (in terms of the model output parameter of interest) is exceeded in a specific area. All the $\pi_{i, j, k, r}$ data have to be used to define the prior models at node 7 , i.e. the probability of a reaching episode, and at node 8 , i.e. the probability of an overcoming episode, in all the $K_{7}$ areas around the volcano.

As presented in the BET_VH model discussion (Marzocchi et al. 2010) since reaching and overcoming episodes in different areas are not mutually exclusive, each area has a specific Beta distribution at both nodes 7 and 8, for each eruption size class and each vent location. In practice, we have to set the two parameters of the Beta distribution ( $\alpha$ and $\beta$ ) for two nodes in $K_{7}$ areas for $I_{4}$ locations and $J_{5}$ size class different distributions (Marzocchi et al. 2010).

As regards indexes and symbols that follow, they are in common with the companion paper Marzocchi et al. (2010) and have been reported in Tables 1 and 2. The index names are kept the same all over both papers: The letter $i$ always refers to vent location (node 4), $j$ to size class (node 5), $k$ to impacted area (node 7), $s$ to the specific threshold (node 8), and $r$ to a single run of the model, while the index relative to the phenomenon ( $p$ at node 6, see Marzocchi et al. 2010) is omitted since in this paper, we refer to a single phenomenon (tephra fallout; see Table 1).

To set all the Beta distributions in each area, we follow a scheme that resembles the one used to account for past data (e.g. see Marzocchi et al. 2010); the difference is that "real" data are replaced by "fake" data coming from the numerical model. In this case, each run must be properly weighted accounting for the accuracy of the overall model rather than counting as one datum. This is done in order to avoid a decrease in variance in the final estimation provided by the model as a mere consequence of a large number of runs of the model itself. In other words, the variance has to be reduced only by adding new real data and/or by increasing the reliability of the model and/or by using the information derived from other models.

To obtain the analytical relationship between the output of the model and the parameters of the Beta distributions, at first we focus on node 7 , and we refer to the generic $k$-th area, the generic $i$ vent location and the generic $j$ size class. The number of reaching episodes $v_{7 ; i, j}^{(k)}$, i.e. the number of times for which $\pi_{i, j, k, r}>0$, $r=1,2, \ldots, N_{r}$, is given by:

$v_{7 ; i, j}^{(k)}=\sum_{r=1}^{N_{r}} H\left(\pi_{i, j, k, r}\right)$

where $H(x)$ is 1 if $x>0$ and 0 elsewhere. Note that $v_{7 ; i, j}^{(k)}$ represents the number of "successes" on the $N_{r}$ "trials".

As mentioned before, each run output has a weight that accounts for the reliability of the model, i.e.

$w_{7}=\frac{\Lambda_{m}}{N_{r}}$

where $\Lambda_{m}$ is the weight of the model measured in terms of "equivalent number of data". In few words, such a number measure the degree of confidence we assign to the model (greater the number, greater the confidence), from a minimum of 1 (largest variance). For a more detailed discussion, see Marzocchi et al. (2004, 2008 and 2010). As we will show later, the weight given by Eq. 9 ensures that the variance of the prior distribution is controlled only by $\Lambda_{m}$ and does not depend on $N_{r}$.

The parameters of the beta distribution at node 7 are then defined as

$\alpha_{7 ; i, j}^{(k)}=\bar{\alpha}_{7 ; i, j}^{(k)}+w_{7} v_{7 ; i, j}^{(k)}$

$\beta_{7 ; i, j}^{(k)}=\bar{\beta}_{7 ; i, j}^{(k)}+w_{7}\left(N_{r}-v_{7 ; i, j}^{(k)}\right)$

where $\bar{\alpha}_{7 ; i, j}^{(k)}$ and $\bar{\beta}_{7 ; i, j}^{(k)}$ represent the parameters of a sort of hyper-prior Beta distribution. Such a hyperprior distribution may represent our very first order guess. A possibility is to base such a hyper-prior Beta distribution on empirical observations in other volcanoes (see, for instance, Tables 2 and 3 in Newhall and Hoblitt 2002), whenever such generalization is assumed to hold. Alternatively, we can set $\bar{\alpha}_{7 ; i, j}^{(k)}=\bar{\beta}_{7 ; i, j}^{(k)}=1$ that would mimic the least informative starting hypothesis (see discussion in Marzocchi et al. 2004, 2008, 2010).

With these definitions, we can now evaluate the total weight of the information brought by the theoretical 
model in terms of equivalent number of data (see Marzocchi et al. 2010).

$\Lambda_{7 ; i, j}^{(k)}=\alpha_{7 ; i, j}^{(k)}+\beta_{7 ; i, j}^{(k)}-1=\bar{\alpha}_{7 ; i, j}^{(k)}+\bar{\beta}_{7 ; i, j}^{(k)}-1+\Lambda_{m}$

that is the number of equivalent data of the hyperprior plus the number of equivalent data of the model. The average probability of this Beta (e.g. Gelman et al. 1995) is

$$
\begin{aligned}
\Theta_{7 ; i, j}^{(k)}= & \frac{\alpha_{7 ; i, j}^{(k)}}{\alpha_{7 ; i, j}^{(k)}+\beta_{7 ; i, j}^{(k)}} \\
= & \frac{\bar{\alpha}_{7 ; i, j}^{(k)}}{\bar{\alpha}_{7 ; i, j}^{(k)}+\bar{\beta}_{7 ; i, j}^{(k)}}\left\{\frac{\bar{\alpha}_{7 ; i, j}^{(k)}+\bar{\beta}_{7 ; i, j}^{(k)}}{\bar{\alpha}_{7 ; i, j}^{(k)}+\bar{\beta}_{7 ; i, j}^{(k)}+\Lambda_{m}}\right\} \\
& +\frac{\nu_{7 ; i, j}^{(k)}}{N_{r}}\left\{\frac{\Lambda_{m}}{\bar{\alpha}_{7 ; i, j}^{(k)}+\bar{\beta}_{7 ; i, j}^{(k)}+\Lambda_{m}}\right\}
\end{aligned}
$$

that represents the weighted average between the hyper-prior average $\left(\frac{\bar{\alpha}_{7: i, j}^{(k)}}{\bar{\alpha}_{7 ; i, j}^{(k)}+\bar{\beta}_{7 ;, j}^{(k)}}\right)$ and the observed frequency of reaching episodes in the $N_{r}$ realizations of the model $\left(\frac{\nu_{7: i, j}^{(k)}}{N_{r}}\right)$; the weights are the relative strengths of the two pieces of information. The parameters $\Theta_{7 ; i, j}^{(k)}$ and $\Lambda_{7 ; i, j}^{(k)}$ completely set the prior distribution parameters for node 7 and represent the input parameters of the BET_VH model (see Marzocchi et al. 2010).

For node 8 , in analogy with node 7 , we set the number of overcoming episodes, i.e. the number of times $\pi_{i, j, k, r}>s$, and the weight of the single datum coming from one model realization as

$v_{8 ; i, j, k}^{(s)}=\sum_{j=1}^{N_{r}} H\left(\pi_{i, j, k, r}-s\right)$

$w_{8}=\frac{\Lambda_{m}}{v_{7 ; i, j}^{(k)}}$

Consequently, the parameters of the beta distribution at node 8 will be

$\alpha_{8 ; i, j, k}^{(s)}=\bar{\alpha}_{8 ; i, j, k}^{(s)}+w_{8} v_{8 ; i, j, k}^{(s)}$

$\beta_{8 ; i, j, k}^{(s)}=\bar{\beta}_{8 ; i, j, k}^{(s)}+w_{8}\left(v_{7 ; i, j}^{(k)}-v_{8 ; i, j, k}^{(s)}\right)$

where $\bar{\alpha}_{8 ; i, j, k}^{(s)}$ and $\bar{\beta}_{8 ; i, j, k}^{(s)}$ represent the parameters of a sort of hyper-prior Beta distribution at node 8 and may be set both at 1 (least informative starting hypothesis). Note that at node 8 since $v_{7 ; i, j}^{(k)}$ may be 0 , Eq. 15 may be not defined. In this case, since from the definitions we have that

$v_{7 ; i, j}^{(k)} \geq v_{8 ; i, j, k}^{(s)} \geq 0$

when $v_{7 ; i, j}^{(k)}=0$, also $v_{8 ; i, j, k}^{(s)}$ is equal to 0 . In this case, the model does not bring any information (see Eqs. 16 and 17), therefore we set

$\alpha_{8 ; i, j, k}^{(s)}=\bar{\alpha}_{8 ; i, j, k}^{(s)}$

$\beta_{8 ; i, j, k}^{(s)}=\bar{\beta}_{8 ; i, j, k}^{(s)}$

Proceeding in analogy with node 7 and accounting for the case $v_{7 ; i, j}^{(k)}=0$, we finally obtain the parameters of prior distributions at node 8 , i.e. the input parameters for BET_VH:

$$
\begin{aligned}
& \Lambda_{8 ; i, j, k}^{(s)}= \begin{cases}\bar{\alpha}_{8 ; i, j, k}^{(s)}+\bar{\beta}_{8 ; i, j, k}^{(s)}-1+\Lambda_{m} & \text { if } v_{7 ; i, j}^{(k)}>0 \\
\alpha_{8 ; i, j, k}^{(s)}+\bar{\beta}_{8 ; i, j, k}^{(s)}-1 & \text { if } \nu_{7 ; i, j}^{(k)}=0\end{cases} \\
& \Theta_{8 ; i, j, k}^{(s)}
\end{aligned}
$$

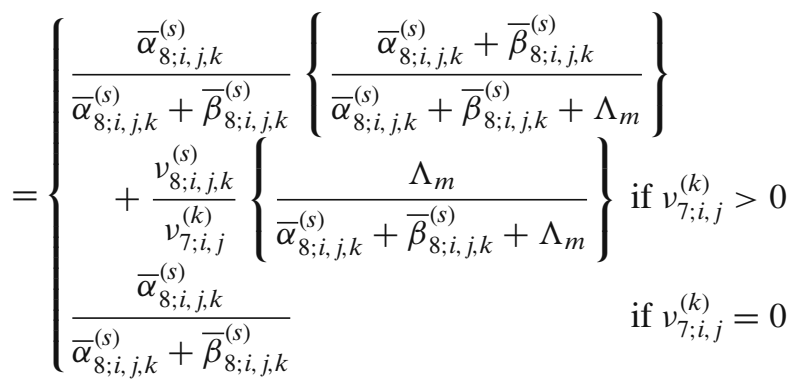

The interpretation of $\Lambda_{8 ; i, j, k}^{(s)}$ and $\Theta_{8 ; i, j, k}^{(s)}$ is the same as at node 7 . The only difference worth being noted is that if in a specific area the phenomenon is never recorded in the $N_{r}$ simulations (i.e. $v_{7 ; i, j}^{(k)}=0$ ), we completely rely on the hyper-prior distribution given by $\bar{\alpha}_{8 ; i, j, k}^{(s)}$ and $\bar{\beta}_{8 ; i, j, k}^{(s)}$. Obviously, in this case, the variance of the distribution is not decreased by the model since it is not informative.

The only parameter that is not set by the model runs is $\Lambda_{m}$, i.e. the equivalent number of data to be assigned to the model. We stress that $\Lambda_{m}$ must be set depending on the (subjective) reliability that the researcher gives to the modeling procedure adopted, i.e. not only to the model itself but also the underlying assumptions and the capability of the $N_{r}$ realizations to successfully describe the whole physical system (e.g. for tephra fall, wind variability, emission rates variability during the eruption, DEM models etc.).

We remark that the use of a hyper-prior distribution has two main interesting consequences. First, if properly chosen, it eliminates areas with "zero" probability, i.e. areas never reached by models' runs: in the cases 
for which $v_{7 ; i, j}^{(k)}=0$, in Eq. 10, we have $\alpha_{7 ; i, j}^{(k)}=\bar{\alpha}_{7 ; i, j}^{(k)}$, meaning that the Beta distribution has average $>0$ if, and only if, $\bar{\alpha}_{7 ; i, j}^{(k)}>0$ (see, e.g. Gelman et al. 1995). The second and maybe the most important consequence is that it allows different models to be applied recursively; specifically, the Beta parameters $\alpha$ and $\beta$ resulting from one model become the hyper-prior parameters for the new model: At node 7, the hyper-prior parameters $\bar{\alpha}_{7 ; i, j}^{(k)}$ and $\bar{\beta}_{7 ; i, j}^{(k)}$ for the second model will be set equal to $\alpha_{7 ; i, j}^{(k)}$ and $\beta_{7 ; i, j}^{(k)}$ from Eqs. 10 and 11 for the first model; in analogy, at node 8 , the hyper-prior parameters $\bar{\alpha}_{8 ; i, j, k}^{(s)}$ and $\bar{\beta}_{8 ; i, j, k}^{(s)}$ for the second model will be set equal to $\alpha_{8 ; i, j, k}^{(s)}$ and $\beta_{8 ; i, j, k}^{(s)}$ from equations of (16) and (17) for the first model; the same procedure can be applied for a third model and so on.

Finally, note that it is not necessary that all models cover the whole set of possible ESs, i.e. all the possible combinations of $i$ and $j$. Obviously, the consistency of the hazard estimation is guaranteed only if at least one model covers all the possible ESs.

\section{References}

Barberi F, Macedonio G, Pareschi MT, Santacroce R (1990) Mapping the tephra fallout risk: an example from Vesuvius (Italy). Nature 344:142-144

Barberi F, Principe C, Rosi M, Santacroce R (1995) Scenario dell'evento eruttivo massimo atteso al Vesuvio in caso di riattivazione a breve-medio termine (Aggiornamento al 20.1.1995). CNR, Gruppo Nazionale per la Vulcanologia, Confidential Report to DPC, $19 \mathrm{pp}$

Barberi F, Davis MS, Isaia R, Nave R, Ricci T (2008) Volcanic risk perception in the Vesuvius population. J Volcanol Geotherm Res 172:244-258

Bonadonna C, Costa A (2010) Modeling of tephra sedimentation from volcanic plumes. In: Fagents SA, Gregg TKP, Lopes RMC (eds) Modeling volcanic processes: the physics and mathematics of volcanism (in press)

Bonadonna C, Macedonio G, Sparks RSJ (2002) Numerical modelling of tephra fallout associated with dome collapses and vulcanian explosions: application to hazard assessment on Montserrat. In: Druitt TH, Kokelaar BP (eds) The eruption of Soufrire Hills Volcano, Montserrat, from 1995 to 1999. Geol Soc London Mem 21. Geological Society, London, pp 517-537

Cioni R, Longo A, Macedonio G, Santacroce R, Sbrana A, Suplizio R, Andronico D (2003) Assessing pyroclastic fall hazard through field data and numerical simulations: example from Vesuvius. J Geophys Res 108:2063. doi:10.1029/ 2001JB000642
Costa A, DellErba F, Di Vito MA, Isaia R, Macedonio G, Orsi G, Pfeiffer T (2009) Tephra fallout hazard assessment at the Campi Flegrei caldera (Italy). Bull Volcanol 71:259-273. doi:10.1007/s00445-008-0220-3

DPC (Dipartimento Protezione Civile) (1995) Pianificazione Nazionale d'Emergenza dell'Area Vesuviana. Dipartimento della Protezione Civile, Roma

DPC/INGV Project V3 (2004-2006) Research on active volcanoes, precursors, scenarios, hazard and risk. Sub-Project V3_2 Campi Flegrei

Gelman A, Carlin JB, Stern HS, Rubin DB (1995) Bayesian data analysis. CRC, Boca Raton

Krishnamurti TN, Kishtawal CM, Zhang Z, et al (2000) Multimodel ensemble forecasts for weather and seasonal climate. J Clim 13:4196-4216

Lindsay J, Marzocchi W, Jolly G, Constantinescu R, Selva J, Sandri L (2010) Towards real-time eruption forecasting in the Auckland volcanic field: testing of BET_EF during the New Zealand National Disaster Exercise 'Ruaumoko'. Bull Volcanol 72:185-204. doi:10.1007/s00445009-0311-9

Macedonio G, Costa A, Longo A (2005) A computer model for volcanic ash fallout and assessment of subsequent hazard. Comput Geosci 31:837-845

Macedonio G, Costa A, Folch A (2008) Ash fallout at Vesuvius: numerical simulations and implication for hazard assessment. J Volcanol Geotherm Res 178:366-377

Marzocchi W, Sandri L, Selva J (2008) BET_EF: a probabilistic tool for long- and short-term eruption forecasting. Bull Volcanol 70:623-632. doi:10.1007/s00445-007-0157-y

Marzocchi W, Sandri L, Selva J (2010) BET_VH: a probabilistic tool for long-time volcanic hazard assessment. Bull Volcanol. doi:10.1007/s00445-010-0357-8

Marzocchi W, Sandri L, Gasparini P, Newhall C, Boschi E (2004) Quantifying probabilities of volcanic events: the example of volcanic hazard at Mount Vesuvius. J Geophys Res 109:B11201. doi:10.1029/2004JB003155

Newhall CG, Hoblitt RP (2002) Constructing event trees for volcanic crises. Bull Volcanol 64:3-20

Orsi G, Di Vito MA, Isaia R (2004) Volcanic hazard assessment at the restless Campi Flegrei caldera. Bull Volcanol 66:514 530. doi:10.1007/s00445-003-0336-4

Orsi G, di Vito MA, Selva J, Marzocchi W (2009) Long-term forecast of eruption style and size at Campi Flegrei caldera (Italy). Earth Planet Sci Lett 287:265-276

Pfeiffer T, Costa A, Macedonio G (2005) A model for the numerical simulation of tephra fall deposits. J Volcanol Geotherm Res 140:273-294

Sandri L, Guidoboni E, Marzocchi W, Selva J (2009) Bayesian Event Tree for Eruption Forecasting (BET_EF) at Vesuvius, Italy: a retrospective forward application to 1631 eruption. Bull Volcanol 71:729-745. doi:10.1007/ s00445-008-0261-7

Selva J, Di Vito M, Marzocchi W, Orsi G, Quaglino M, Sandri L (2007) Probability map of vent opening at Campi Flegrei, Italy. In: Abstracts of the IUGG XXIV general assembly, 2-13/7/2007, Perugia (Italy) (Asbtract number VS008 6778)

Simkin T, Siebert L (1994) Volcanoes of the world. Geoscience Press, Tucson 